\title{
Phonon excitation by electron beams in nanographenes
}

\author{
J. R. M. Saavedra ${ }^{1}$ and F. Javier García de Abajo ${ }^{1,2, *}$ \\ ${ }^{1}$ ICFO-Institut de Ciencies Fotoniques, The Barcelona Institute of Science and Technology, 08860 Castelldefels (Barcelona), Spain \\ ${ }^{2}$ ICREA-Institució Catalana de Recerca i Estudis Avançats, Passeig Lluís Companys 23, 08010 Barcelona, Spain
}

(Received 24 July 2015; published 30 September 2015)

\begin{abstract}
Electron beams have the ability of exciting vibrational modes (phonons) in molecules and nanoclusters, which can be currently probed with atomic spatial resolution through electron energy-loss spectroscopy (EELS). This scenario is similar to the excitation of plasmons with light, except that electrons allow much tighter spatial focusing. Additionally, phonons possess larger quality factors and lifetimes. In an effort to exploit these appealing properties, we theoretically investigate the interaction of focused electron beams with the vibrational modes of carbon nanographenes. The EELS probability is found to mimic the density of vibrational states, both of which evolve smoothly with cluster size towards the limit of extended graphene. Our results support the use of phonons as long-lived, tightly confined substitutes of plasmons, combined with electron-beam-mediated mode-selective excitation.
\end{abstract}

DOI: 10.1103/PhysRevB.92.115449

PACS number(s): 79.20.Uv, 63.22.Kn, 63.22.Rc, 81.07.Nb

\section{INTRODUCTION}

The ability of manipulating light and electrons at ever faster speeds over increasingly smaller distances is central for many advances in information and sensing technologies. In this respect, plasmons - the collective electron oscillations of conduction electrons in metals-possess several appealing properties (robustness, large optical interaction, and tight spatial focusing down to the nanometer scale) that make them attractive for such technologies [1-3]. A large deal of work is currently being devoted to exploiting the quantum aspects of plasmons for information processing [4,5], as well as the field confinement and enhancement associated with these excitations for sensing [6-8] and catalysis $[9,10]$, among other feats. Unfortunately, inelastic losses produce a dramatic reduction in the plasmon lifetime down to a few optical cycles, thus limiting their range of application. Atomic vibrations in molecular-scale structures share some of the plasmon properties, combined with larger lifetimes and smaller spatial features. Finding the means of controllably exciting and manipulating phonons at subnanometer distances is therefore important to exploit their potential for technology.

The answer to this problem could rely on recent advances in electron microscopy. It is now possible to use tightly focused (subnanometer) electron beams for probing sample excitations with $<10 \mathrm{meV}$ energy resolution [11]. This combination of spatial and spectral resolutions enables the excitation and monitoring of phonons with atomic precision, which can be exploited from a fundamental viewpoint for understanding atomic vibrations in small structures, but also with a view to applications, using electrons for on-demand creation and manipulation of those modes. A recent theoretical analysis shows that fast electrons can actually interact with localized vibrations of diatomic molecules [12].

Here, we theoretically investigate the potential of electron beams for exciting and manipulating phonons. The interaction between fast electrons and phonons is described through a quantum-mechanical formalism that we use to study their

*javier.garciadeabajo@icfo.es signatures in electron energy-loss spectroscopy (EELS). We predict relatively large EELS probabilities, which appear to be closely related to the local density of vibrational states (LDVS). Our calculations suggest that the spatial distribution associated with different modes can be used to selectively excite specific phonons. These are essential elements for the application of atomic-scale vibrations in future signalprocessing devices.

\section{CALCULATION OF THE EELS PROBABILITY}

The general theory of inelastic scattering of fast electrons and EELS is well established and described in previous works [13-16]. Here, we derive a Hamiltonian formalism capable of describing the inelastic interaction of fast electrons with the vibrations of an atomic cluster, focusing for simplicity on nanographene islands that range in size from small polycyclic aromatic hydrocarbons to larger structures containing several thousand atoms. The equivalence of this formalism and the standard formulation of EELS [16] is discussed in the Appendix. More precisely, we describe phonons within a second-quantization formalism, while the electron is treated as a classical external potential, neglecting retardation effects for simplicity. We analyze the electron-phonon interaction through the Hamiltonian

$$
H=\sum_{n}\left[\hbar \omega_{n} b_{n}^{+} b_{n}+g_{n}(t)\left(b_{n}^{+}+b_{n}\right)\right],
$$

where $n$ runs over the vibrational modes, which we refer to as phonons in what follows, while $b_{n}\left(b_{n}^{+}\right)$annihilates (creates) a quantum of mode $n$. Neglecting retardation, the interaction between the electron and the phonon mode $n$ is described by the coupling energy

$$
g_{n}(t)=-e \int d^{3} \mathbf{r} \frac{\rho_{n}(\mathbf{r})}{\left|\mathbf{r}-\mathbf{r}_{\mathrm{e}}(t)\right|}
$$

(i.e., the electrostatic energy due to the one-phonon charge in the presence of the electron), where $\mathbf{r}_{\mathrm{e}}(t)$ is the electron trajectory and $\rho_{n}(\mathbf{r})$ is the perturbed charge density of the island associated with mode $n$. The time dependence in $g_{n}(t)$ comes from the electron, which we assume to move with constant 
velocity $v$ along a direction normal to the graphene plane. This type of Hamiltonian has been previously used to describe EELS associated with graphene plasmons [17].

The phonon charge is obtained from the carbon atom displacements ( $\mathbf{u}_{l}^{n}$, where $l$ labels carbon atoms) from the equilibrium positions $\left(\mathbf{R}_{l}\right)$ associated with one quantum of vibration, assuming a rigid motion of the atomic electron charge density. More precisely,

$$
\rho_{n}(\mathbf{r})=\sum_{l}\left[\rho^{\mathrm{C}}\left(\mathbf{R}-\mathbf{R}_{l}-\mathbf{u}_{l}^{n}, z\right)-\rho^{\mathrm{C}}\left(\mathbf{R}-\mathbf{R}_{l}, z\right)\right],
$$

where $\rho^{\mathrm{C}}(\mathbf{r})$ is the charge density of one of the carbon atoms and we use the notation $\mathbf{r}=(\mathbf{R}, z)$. For simplicity, we assume the carbon cluster to be planar, with all atoms lying in the $z=0$ plane, and we neglect the interaction with hydrogens that typically passivate the edges of the structure. The atomic charge density $\rho^{\mathrm{C}}(\mathbf{r})$ includes both electrons and nuclear charges, and we further approximate it from the density of the free-space atom [18] (i.e., neglecting variations due to the rearrangement of valence electrons in the material). In Eq. (2), the atomic displacements correspond to the normal modes of the system, which satisfy the orthogonality relation $\sum_{l} \mathbf{u}_{l}^{n}$. $\mathbf{u}_{l}^{n^{\prime}}=\left(\hbar / 2 M \omega_{n}\right) \delta_{n n^{\prime}}$. Notice that this expression incorporates a normalization of the displacement vectors corresponding to singly occupied phonon modes, with $M$ representing the carbon atom mass. In practice, we obtain the normal mode amplitudes and frequencies $\left(\mathbf{u}_{l}^{n}\right.$ and $\left.\omega_{n}\right)$ from a simple model that only accounts for the C-C stretching energy [19].

For the relatively low excitation probabilities encountered in electron microscope spectroscopies [16], it is safe to describe phonon excitations by the beam electrons within first-order perturbation theory. We assume the sample to be initially prepared in the ground state. Each phonon mode $n$ can then be described independently, so the probability of exciting it during the finite time of interaction with the electron is obtained by integrating the Schrödinger equation. We find the final amplitude of mode $n$ to be [17]

$$
\xi_{n}=\frac{-i}{\hbar} \int_{-\infty}^{\infty} d t e^{i \omega_{n} t} g_{n}(t)
$$

corresponding to a probability $\left|\xi_{n}\right|^{2}$ of undergoing an energy loss $\hbar \omega_{n}$. This allows us to write the EELS probability distribution as

$$
\Gamma^{\mathrm{EELS}}(\omega)=\frac{\gamma}{2 \pi} \sum_{n} \frac{\left|\xi_{n}\right|^{2}}{\left(\omega-\omega_{n}\right)^{2}+(\gamma / 2)^{2}},
$$

where a phenomenological Lorentzian broadening $\hbar \gamma$ is introduced to represent the full width at half maximum of the zero-loss peak (ZLP, $10 \mathrm{meV}$ throughout this work), which is large in practice compared with the intrinsic phonon width. Notice that the definition of $\Gamma^{\operatorname{EELS}}(\omega)$ in Eq. (4) yields units of inverse frequency, so that the probability is normalized per unit of frequency-loss range.

The time integral in Eq. (3) can be readily carried out for an electron moving along $z$ (normal to the carbon plane) with velocity $v$ [i.e., $\left.\mathbf{r}_{\mathrm{e}}(t)=\left(\mathbf{R}_{\mathrm{e}}, v t\right)\right]$ to yield

$$
\xi_{n}=\frac{2 i e}{v \hbar} \int d^{3} \mathbf{r} e^{i \omega_{n} z / v} \rho_{n}(\mathbf{r}) K_{0}\left(\omega_{n}\left|\mathbf{R}-\mathbf{R}_{\mathrm{e}}\right| / v\right) .
$$

Notice that the customary modified Bessel functions $K_{m}$ of orders $m=0$ and $m=1$ appear throughout this analysis. Inserting Eq. (2) into this expression, we find

$$
\xi_{n}=\frac{2 i e}{v \hbar} \sum_{l}\left[F_{n}\left(\mathbf{R}_{\mathrm{e}}-\mathbf{R}_{l}-\mathbf{u}_{l}^{n}\right)-F_{n}\left(\mathbf{R}_{\mathrm{e}}-\mathbf{R}_{l}\right)\right],
$$

where

$$
F_{n}(\mathbf{R})=\int d^{3} \mathbf{r}^{\prime} e^{i \omega_{n} z^{\prime} / v} \rho^{\mathrm{C}}\left(\mathbf{r}^{\prime}\right) K_{0}\left(\omega_{n}\left|\mathbf{R}^{\prime}-\mathbf{R}\right| / v\right) .
$$

We can now Taylor-expand Eq. (5) in powers of the displacements $\mathbf{u}_{l}^{n}$, which we assume to be small compared with the equilibrium interatomic distances. Retaining only linear terms, one finds

$$
\xi_{n} \approx \frac{2 i e}{v \hbar} \sum_{l} \mathbf{u}_{l}^{n} \cdot \mathbf{G}_{n}\left(\mathbf{R}_{\mathrm{e}}-\mathbf{R}_{l}\right)
$$

where

$\mathbf{G}_{n}(\mathbf{R})=\frac{\omega_{n}}{v} \int d^{2} \mathbf{R}^{\prime} \tilde{\rho}^{\mathrm{C}}\left(\mathbf{R}^{\prime}\right) K_{1}\left(\omega_{n}\left|\mathbf{R}-\mathbf{R}^{\prime}\right| / v\right) \frac{\mathbf{R}-\mathbf{R}^{\prime}}{\left|\mathbf{R}-\mathbf{R}^{\prime}\right|}$

and we have introduced the $z$-integrated atomic charge density

$$
\tilde{\rho}^{\mathrm{C}}(\mathbf{R})=\int_{-\infty}^{\infty} d z e^{i \omega_{n} z / v} \rho^{\mathrm{C}}(\mathbf{R}, z) .
$$

Now, for the typical acceleration voltages used in transmission electron microscopes (i.e., $80-300 \mathrm{kV}$, corresponding to $v / c \approx$ $0.50-0.78$ ) and for phonon energy losses $\hbar \omega_{n} \leqslant 0.2 \mathrm{eV}$ (i.e., the upper limit in carbon allotropes), we have $v / \omega_{n}>500 \mathrm{~nm}$, which is a large distance compared with the sizes of the atomic clusters under consideration. In particular, the argument in the exponential of Eq. (8) is small over the region in which $\rho^{\mathrm{C}}$ takes nonnegligible values, and therefore, we can approximate

$$
\tilde{\rho}^{\mathrm{C}}(\mathbf{R}) \approx \int_{-\infty}^{\infty} d z \rho^{\mathrm{C}}(\mathbf{R}, z) .
$$

Likewise, we can apply the small argument limit of the Bessel function $K_{1}(\theta) \approx 1 / \theta$, which permits approximating Eq. (7) as

$$
\mathbf{G}_{n}(\mathbf{R}) \approx \int \frac{d^{2} \mathbf{R}^{\prime}}{R^{\prime}} \tilde{\rho}^{\mathrm{C}}\left(\mathbf{R}-\mathbf{R}^{\prime}\right) \hat{\mathbf{R}}^{\prime}
$$

It is now convenient to express the atomic charge as $\rho^{\mathrm{C}}(\mathbf{r})=$ $e[Z \delta(\mathbf{r})-n(\mathbf{r})]$ in terms of the atomic electron density $n(\mathbf{r})$ and the nuclear charge $e Z$. Writing the integral in polar coordinates, we finally find

$$
\mathbf{G}_{n}(\mathbf{R})=e\left[\frac{Z}{R}-2 \int_{0}^{\pi} d \varphi \cos \varphi \int_{0}^{\infty} d R^{\prime} \tilde{n}(d)\right] \hat{\mathbf{R}},
$$

where $d=\sqrt{R^{2}+R^{\prime 2}-2 R R^{\prime} \cos \varphi}$ and we use $\tilde{n}(\mathbf{R})=$ $\int d z n(\mathbf{r})$ by analogy to Eq. (9).

In what follows, we show results obtained from Eqs. (4), (6), and (10). For simplicity, we neglect the spatial extension of $1 s$ electrons in carbon, so we effectively have $Z=4$ and approximate $n(\mathbf{r})=2\left|\psi_{2 s}(r)\right|^{2}+\left|\psi_{2 p}(r)\right|^{2}\left(R^{2} / 2+z^{2}\right) / r^{2}$; that is, we take two electrons in the normalized $\psi_{2 s}$ orbital, one electron shared in the in-plane $(x / r) \psi_{2 p}$ and $(y / r) \psi_{2 p}$ orbitals, and one valence electron in the out-of-plane $(z / r) \psi_{2 p}$ orbital. In practice, we use tabulated atomic data for these orbitals [18]. 


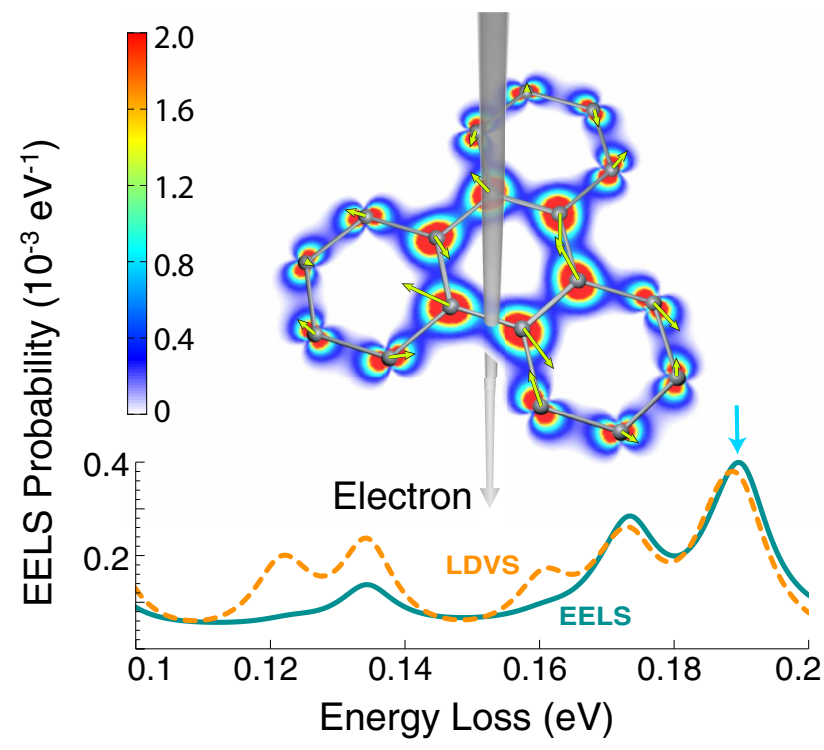

FIG. 1. (Color online) Spatially resolved excitation of phonons in triphenylene. We show an EELS spectrum (solid curve) calculated for $100 \mathrm{keV}$ electrons focused at the position indicated by a vertical arrow in the density plot. The latter represents a $0.19 \mathrm{eV}$ energy filtered map. Atomic displacements associated with one of the 4-fold degenerate $0.19 \mathrm{eV}$ vibrational modes are shown by open arrows. The dashed curve represents the local density of vibrational states (LDVS) at the beam position (see Sec. III).

Incidentally, the same results are obtained by treating the carbon cluster as a classical array of coupled particles with a charge density $\rho^{\mathrm{C}}(\mathbf{r})$, linearizing their displacements, and calculating the EELS probability from the field induced back at the electron (Eq. (8) of Ref. [16]).

It is important to note that for sufficiently energetic beams the electron velocity $v$ enters this formalism only through a factor $1 / v$ in the transition amplitude $\xi_{n}$ (i.e., the EELS probability scales as $\left.1 / v^{2}\right)$, so although the results presented below are calculated for $100 \mathrm{keV}$ electrons $(v \approx 0.55 c)$, it is straightforward to apply them to other electron energies (e.g., the plotted intensities have to be multiplied by a factor of $\approx 77$ when considering $1 \mathrm{keV}$ electrons).

An illustrative example of phonon-related EELS probabilities is presented in Fig. 1 for electrons interacting with a triphenylene molecule. The spectrum, which corresponds to the electron trajectory depicted in the inset, displays several intense features associated with different vibrational modes. In particular, the highest-energy peak involves the excitation of 4-fold degenerate combinations of localized stretching modes, with atomic displacements as shown by arrows in the inset for one of them. The inset also shows a contour plot with the loss probability at this energy loss as a function of electron beam position, suggesting a confinement towards the central ring of the molecule.

When examining atomically resolved phonon excitations, it is important to consider the finite size of the electron beam. Assuming a large aperture in the electron analyzer, so that all the inelastic signal is collected, the EELS probability is simply obtained from the average of the above results (assuming a linelike beam) over different positions, weighted by the beam

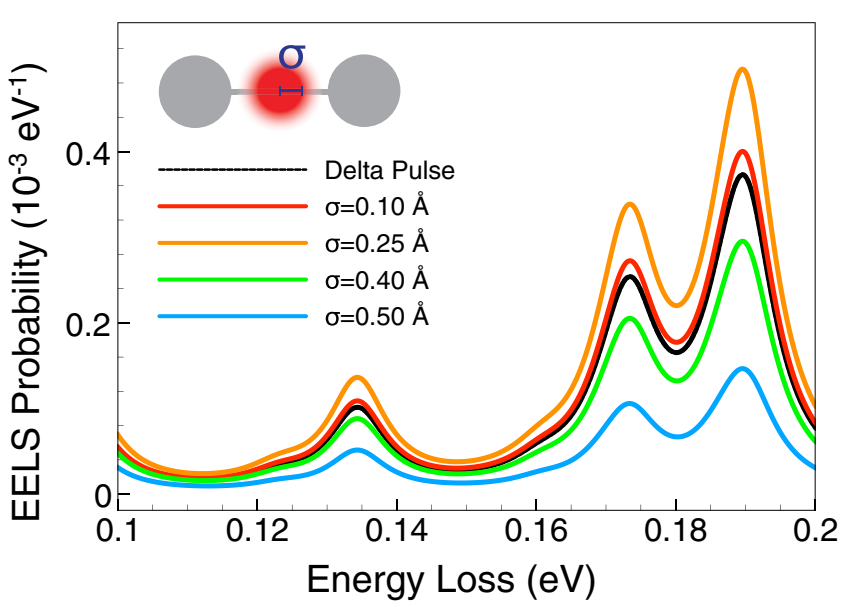

FIG. 2. (Color online) Effect of finite electron beam lateral size. We show the EELS probability under the same conditions as in Fig. 1 for different values of the half width at half maximum $\sigma$. The beam has Gaussian profiles.

intensity profile [20]. Some results for Gaussian beams of different half width at half maximum $\sigma$ are shown in Fig. 2, considering the same sample and central position of the beam as in Fig. 1 (i.e., at the center of a C-C bond). It is interesting to note that the probability is initially increasing with beam size, as a larger portion of the electrons is passing closer to the atoms, and eventually decreases for higher $\sigma$ when an even larger portion is too far to excite them. For simplicity, we show results for $\sigma=0$ in what follows, which are actually similar to those obtained for a realistically attainable beam size $2 \sigma \sim 0.5 \AA$ (see Fig. 2).

\section{PROBING THE LOCAL DENSITY OF VIBRATIONAL STATES THROUGH EELS}

It has been argued that EELS in photonic structures yields information on the so-called local density of optical states [21]. Likewise, the losses due to the excitation of vibrational modes can provide insight into the spatial distribution of those modes. A first indication that this is the case is given by comparing the dashed (LDVS) and solid (EELS) curves in Fig. 1. A more detailed analysis of this idea is presented in Fig. 3, where we consider structures of increasing size (top to bottom) and compare the EELS probability for a given beam position and the LDVS at that position. We define the LDVS by analogy to the density of electronic states [22] as the spatially and spectrally resolved distribution of vibrational modes. More precisely, $\rho_{l}^{\mathrm{LDVS}}(\omega) \propto \sum_{n}\left|\mathbf{u}_{l}^{n}\right|^{2} \delta\left(\omega-\omega_{n}\right)$, where $l$ labels atomic positions and $n$ runs over modes. To facilitate a comparison with EELS, we convolute the LDVS with a space Lorentzian, so we actually plot $\left(\delta_{r} / 2 \pi\right) \sum_{l} \rho_{l}^{\text {LDVS }} /\left[\left|\mathbf{R}-\mathbf{R}_{l}\right|^{2}+\left(\delta_{r} / 2\right)^{2}\right]$ with $\delta_{r}=0.2 \mathrm{~nm}$. Additionally, we introduce the same frequency broadening as in the ZLP [see Eq. (4)]. From Fig. 3, we observe a clear resemblance between EELS and LDVS, which is maintained for all clusters considered up to the extended graphene limit (lower panel), although the agreement between both quantities is far from complete. We also examine the full spatial dependence of these magnitudes for selected energies 

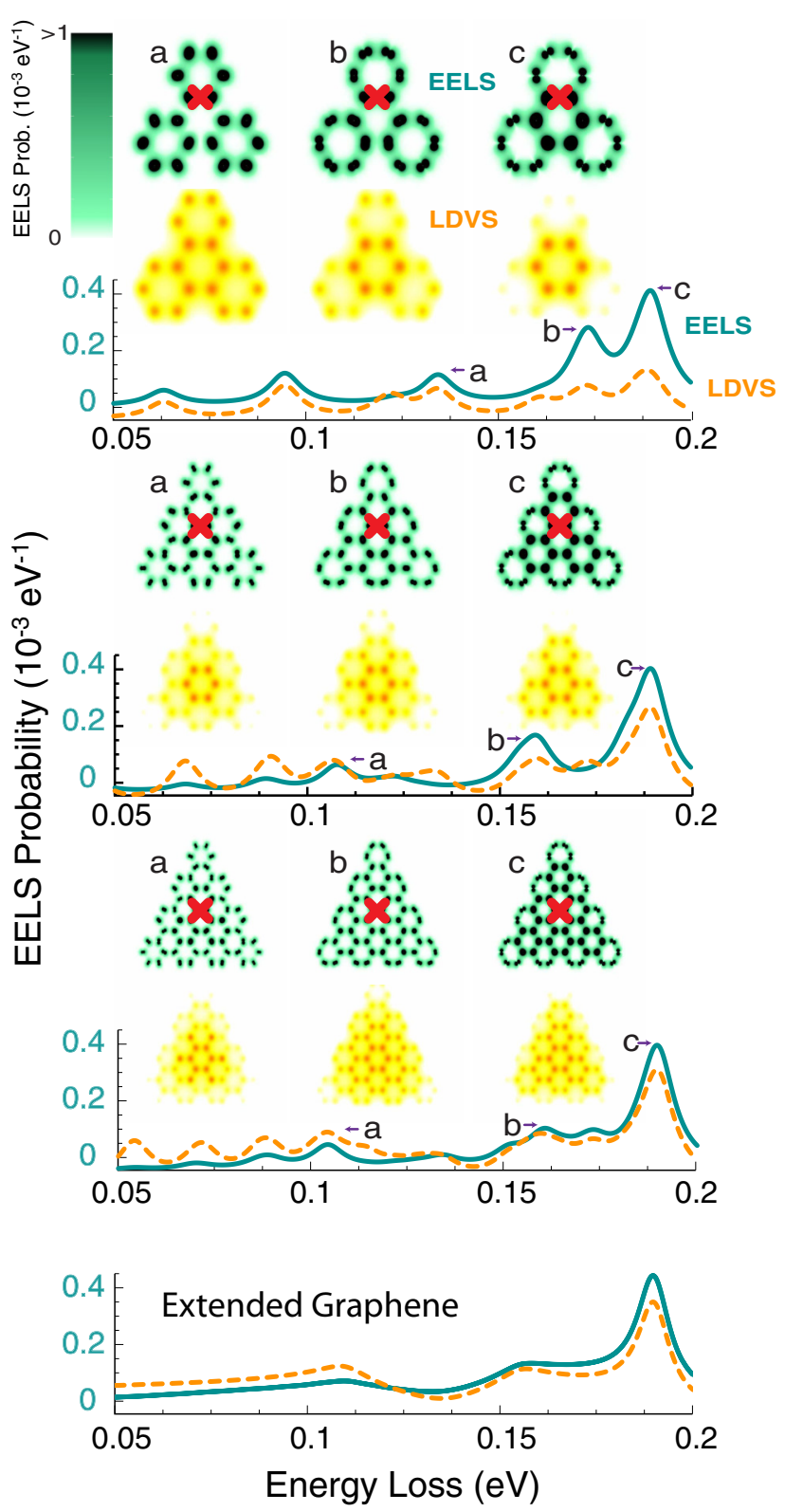

FIG. 3. (Color online) Correlation between EELS and LDVS. EELS spectra (solid curves) and energy-filtered maps (upper insets in each case, corresponding to the labeled spectral features) are given in absolute units (loss probability per eV of energy-loss range) for nanographenes of increasing size (top to bottom), compared with the LDVS (broken curves and lower contour-plot insets, both in arbitrary units). The beam position is indicated by a red cross in the energy-filtered maps.

(see insets) and find that they undergo a comparable degree of localization and display similar spatial patterns.

The distinct spatial distributions of different modes suggest the possibility of selectively exciting them by aiming the electron beam at different locations. Figure 4 shows that this can be achieved with modes of different energy. For example, for the outer beam position under consideration, there is a preferential excitation of the $0.17 \mathrm{eV}$ mode, whereas the innermost trajectory excites the $0.19 \mathrm{eV}$ mode with dominant strength.

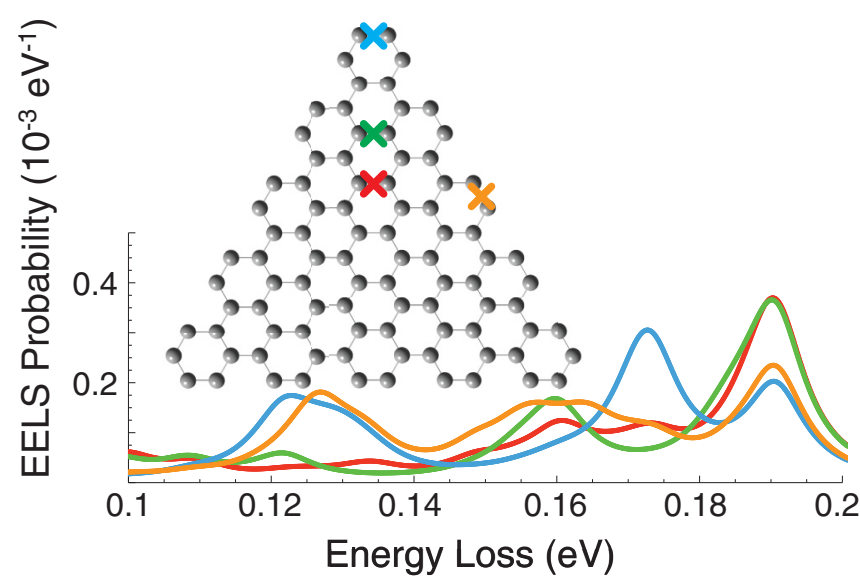

FIG. 4. (Color online) Selective phonon excitation. We show the loss probability for electrons passing at different positions relative to a 90-atom carbon nanotriangle, showing the selective excitation of different modes at each position. The electron positions are indicated by crosses in the upper inset with the same colors as the corresponding spectral curves.

\section{CONCLUSION}

Our results indicate that electron beams undergo large interaction with the vibrational modes of nanographenes, which can then be probed through EELS in state-of-the-art TEMs. The loss probability is in fact closely related to the local density of vibrational states, thus suggesting the use of their mode-dependent spatial distributions to accomplish selective excitation of a specific phonon by focusing the beam at a region of maximum density. This situation is similar to the optical manipulation of plasmons in metallic nanostructures, so phonons are also suitable candidates for signal propagation and manipulation, with the additional advantage of their larger quality factors. We note that rather large excitation and absorption probabilities can be achieved through the use of relatively low energy electrons in the sub-keV range. Nanofocusing of such electrons could be accomplished by using nanoscale field-emission sources (e.g., metal tips) in close proximity to the samples. Our results support the potential of phonons as long-lived substitutes of plasmons in molecular-size structures.

\section{ACKNOWLEDGMENTS}

This work has been supported in part by the the Spanish MINECO (MAT2014-59096-P) and the European Commission (Graphene Flagship CNECT-ICT-604391). J.R.M.S. acknowledges financial support through AGAUR FI_B $00492-$ 2015 .

\section{APPENDIX: EQUIVALENCE OF HAMILTONIAN AND DIELECTRIC APPROACHES TO EELS}

In order to establish a relation between the present Hamiltonian formalism and previous dielectric approaches, it is convenient to write Eq. (1) as $g_{n}(t)=-e \phi_{n}\left[\mathbf{r}_{e}(t)\right]$, where $\phi_{n}(\mathbf{r})=\int d^{3} \mathbf{r}^{\prime} \rho_{n}\left(\mathbf{r}^{\prime}\right) /\left|\mathbf{r}-\mathbf{r}^{\prime}\right|$ is the electric potential due to the charge $\rho_{n}$ associated with the phonon mode 
$n$. Considering now a general external charge $\rho^{\text {ext }}(\mathbf{r}, t)$ instead of the electron, the coupling coefficients become $g_{n}(t)=\int d^{3} \mathbf{r} \rho^{\mathrm{ext}}(\mathbf{r}, t) \phi_{n}(\mathbf{r})$. Following previous work for this type of Hamiltonian [17], this allows us to write the expression (Eq. (37) of Ref. [17])

$$
W^{\text {ind }}\left(\mathbf{r}, \mathbf{r}^{\prime}, \omega\right)=\frac{2}{\hbar} \sum_{n} \frac{\omega_{n} \phi_{n}(\mathbf{r}) \phi_{n}\left(\mathbf{r}^{\prime}\right)}{\omega^{2}-\omega_{n}^{2}+i 0^{2}}
$$

for the induced screened interaction, which is defined such that $\int d^{3} \mathbf{r}^{\prime} W^{\text {ind }}\left(\mathbf{r}, \mathbf{r}^{\prime}, \omega\right) \rho^{\text {ext }}\left(\mathbf{r}^{\prime}, \omega\right)$ is the electric potential at position $\mathbf{r}$ due to $\rho^{\text {ext }}$ in frequency space $\omega$. Equation (A1) can be directly inserted into Eq. (10) of Ref. [16] to yield the loss probability

$$
\Gamma^{\mathrm{EELS}}(\omega)=\sum_{n}\left|\xi_{n}\right|^{2} \delta\left(\omega-\omega_{n}\right)
$$

where $\xi_{n}$ coincides with the definition of Eq. (3). Finally, Eq. (4) is derived from this expression by convoluting it with a finite ZLP frequency width $\gamma$. This result confirms that the present Hamiltonian formalism is equivalent to the dielectric approach that is traditionally used in EELS.
[1] R. Zia, J. A. Schuller, A. Chandran, and M. L. Brongersma, Mater. Today 9(7-8), 20 (2006).

[2] A. Polman, Science 322, 868 (2008).

[3] M. I. Stockman, Phys. Today 64(2), 39 (2011).

[4] D. E. Chang, A. S. Sörensen, P. R. Hemmer, and M. D. Lukin, Phys. Rev. Lett. 97, 053002 (2006).

[5] A. V. Akimov, A. Mukherjee, C. L. Yu, D. E. Chang, A. S. Zibrov, P. R. Hemmer, H. Park, and M. D. Lukin, Nature (London) 450, 402 (2007).

[6] H. Xu, E. J. Bjerneld, M. Käll, and L. Börjesson, Phys. Rev. Lett. 83, 4357 (1999).

[7] K. Kneipp, Y. Wang, H. Kneipp, L. T. Perelman, I. Itzkan, R. R. Dasari, and M. S. Feld, Phys. Rev. Lett. 78, 1667 (1997).

[8] S. Nie and S. R. Emory, Science 275, 1102 (1997).

[9] Z. W. Seh, S. Liu, M. Low, S.-Y. Zhang, Z. Liu, A. Mlayah, and M.-Y. Han, Adv. Mater. 24, 2310 (2012).

[10] C. Clavero, Nat. Photon. 8, 95 (2014).

[11] O. L. Krivanek, T. C. Lovejoy, N. Dellby, T. Aoki, R. W. Carpenter, P. Rez, E. Soignard, J. Zhu, P. E. Batson, M. J. Lagos et al., Nature (London) 514, 209 (2014).
[12] C. Dwyer, Phys. Rev. B 89, 054103 (2014).

[13] R. F. Egerton, Electron Energy-Loss Spectroscopy in the Electron Microscope (Plenum Press, New York, 1996).

[14] P. M. Echenique, J. Bausells, and A. Rivacoba, Phys. Rev. B 35, 1521 (1987).

[15] F. Ouyang and M. Isaacson, Ultramicroscopy 31, 345 (1989).

[16] F. J. García de Abajo, Rev. Mod. Phys. 82, 209 (2010).

[17] F. J. García de Abajo, ACS Nano 7, 11409 (2013).

[18] E. Clementi and C. Roetti, At. Data Nucl. Data Tables 14, 177 (1974).

[19] S. Viola Kusminskiy, D. K. Campbell, and A. H. Castro Neto, Phys. Rev. B 80, 035401 (2009).

[20] R. H. Ritchie and A. Howie, Philos. Mag. A 58, 753 (1988).

[21] F. J. García de Abajo and M. Kociak, Phys. Rev. Lett. 100, 106804 (2008).

[22] N. W. Ashcroft and N. D. Mermin, Solid State Physics (Harcourt College Publishers, New York, 1976). 